\title{
ORIGINAL
}

\section{BROTES POR NOROVIRUS EN RESIDENCIAS Y CENTROS SANITARIOS DE CATALUÑA}

\author{
Pere Godoy (1,2), Angela Dominguez (2), Josep Alvarez (1), Neus Camps (1), Irene Barrabeig (1), \\ Rosa Bartolomé (3), María Rosa Sala (1), Dolors Ferre (4), Helena Pañella (4), Joan Torres (1), \\ Sofía Minguell (1), Miquel Alsedà (1), Analía Pumares (1) por el grupo de estudio de gastroenteritis \\ víricas en Cataluña.
}

(1) Departamento de Salud de la Generalidad de Cataluña.

(2) CIBER Epidemiología y Salud Pública (CIBERESP).

(3) Servicio de Microbiología Vall d'Hebron Hospital, Catalonia, Spain.

(4) Agencia de Salud Pública de Barcelona.

\section{RESUMEN}

Fundamentos. La baja dosis infectiva y las múltiples vías de transmisión facilitan la presentación de brotes de norovirus. El objetivo fue comparar la incidencia de brotes por norovirus en hospitales y residencias en Cataluña.

Métodos. Se realizó un estudio descriptivo de la serie de brotes de norovirus en el período del 15/10/2004 al 30/10/2005. Se rellenó una encuesta epidemiológica para cada brote. Las variables fueron: número de expuestos, enfermos, mecanismo de transmisión, ámbito (centros sanitarios o residencias), región sanitaria, mes del año, y duración. Mediante técnicas de PCR se investigó la presencia de norovirus en muestras clínicas. Se calculó la incidencia en cada centro y la incidencia anual de brotes por centros. Las diferencias se determinaron con la prueba de $\chi^{2}$ y la t de Student con un grado de significación (p) inferior a 0,05 .

Resultados. Se detectaron 17 brotes, 6 en centros sanitarios y 11 en residencias. La tasa de ataque global fue del $33,4 \%(652 / 1951)$ y fue ligeramente superior en las residencias $(35,2 \%)$ que en los centros sanitarios $(31,4 \%)$.

El 94,1\% (16/17) de los brotes se produjeron por transmisión persona a persona y sólo el 5,9\% (1/17) por alimentos. La media de días entre el primer y último caso del brote fue de $11,4(\mathrm{DE}=6,9)$.

La duración media de los síntomas fue de 2,39 días $(\mathrm{SD}=1,6)$ y también fue superior en los pacientes hospitalizados 2,63 ( $\mathrm{SD}=1,7)$ en comparación a los pacientes de residencias $1,97(\mathrm{SD}=1,7)(\mathrm{p}<0,0001)$.

Conclusiones. Norovirus es responsable de un número importante de brotes por transmisión persona a persona. Se debe protocolizar su control para reducir su número y su duración.

Palabras clave: Brote. Gastroenteritis. Incidencia. Vigilancia. Epidemiología. Norovirus. Norwalk like virus. Hospitales. Residencias. Lavado de manos.

\section{Correspondencia:}

Pere Godoy

Dirección General de Salud Pública

C/ Roc Boronat, 81-95. 08005 Barcelona

Tel: +34 935513583

Correo electrónico: pere.godoy@gencat.net

\section{ABSTRACT}

\section{Norovirus Outbreaks in Hospitals and Nursing Homes in Catalonia, Spain}

Background. The low infectious dose and multiple transmission routes favour the appearance of norovirus outbreaks. The objective of this study was to compare the incidence of norovirus outbreaks in hospitals and nursing homes in Catalonia.

Methods. A descriptive study of norovirus outbreaks between 15/10/2004 and 30/10/2005 was carried out. An epidemiological survey was completed for each outbreak. Norovirus in clinical samples was determined by PCR techniques. The incidence in each centre and the annual incidence of outbreaks by centre were calculated. Differences were calculated using the chi-square test and the Student's $t$ test, taking a $p$ value of $>0.05$ as significant.

Results. Seventeen outbreaks (6 in hospitals and 11 in nursing homes) were detected. The global attack rate was $33.4 \%(652 / 1951)$ and was slightly higher in nursing homes $(35.2 \%)$ than in hospitals $(31.4 \%)$.

A total of $94.1 \%(16 / 17)$ of outbreaks were caused by person-to-person transmission and only $5.9 \%$ (1/17) by foods. The mean number of days between the first and last case was $11.4(\mathrm{SD}=6.9)$.

The mean duration of symptoms was 2.39 days $(\mathrm{SD}=1.6)$, and was higher hospitals, $2.63(\mathrm{SD}=1.7)$, than in nursing homes, $1.97(\mathrm{SD}=1.7)(\mathrm{p}<0.0001)$.

Conclusions. Norovirus is responsible for a large number of outbreaks due to person-to-person transmission. Control should be standardized to reduce the number and duration of outbreaks.

Keywords: Outbreak. Gastroenteritis. Incidence. Surveillance. Epidemiology. Norovirus. Norwalk like virus. Hospital. Residences. Hand washing. 


\section{INTRODUCCIÓN}

El reservorio y la fuente de infección de los norovirus son las personas enfermas y estudios de prevalencia en población general también han demostrado la existencia de infecciones totalmente asintomáticas que podrían tener un papel importante como fuente de infección ${ }^{1}$. Estudios recientes señalan un periodo mediano de eliminación de virus después de una infección experimental de al menos 28 días (rango 13-56 días $)^{2}$. La dosis infecciosa es muy baja, menos de 100 partículas víricas ${ }^{1-4}$, por lo que los Norovirus son también uno de los principales agentes responsables de brotes nosocomiales por transmisión persona a persona entre los residentes y los propios trabajadores sanitarios 5 .

El envejecimiento de la población en las últimas décadas junto con los cambios sociales ha comportado un aumento de la población adulta con patologías crónicas que precisan frecuentes hospitalizaciones y asistencia en centros especializados ${ }^{5}$. Esto ha facilitado la proliferación de hospitales y residencias asistidas dirigidas fundamentalmente a las personas mayores. En estos centros el grado de dependencia de algunas personas es muy elevado lo que puede comportar un riesgo de infecciones nosocomiales que se transmitan a partir de los propios trabajadores de los centros ${ }^{5-7}$. Este riesgo además se puede ver incrementado por la dificultad de aplicar los protocolos para evitar estas infecciones debido a aspectos como las sobrecargas de trabajo o la insuficiente formación de los mismos trabajadores.

También se han descrito, de forma similar a la gripe estacional, aumento de la incidencia de brotes debido a la aparición de nuevas cepas emergentes ante las cuales la mayoría de la población se comporta como susceptible ${ }^{8}$.

El objetivo del estudio fue describir y comparar los de brotes de gastroenteritis por norovirus en hospitales y residencias en Cataluña.

\section{MATERIAL Y MÉTODOS}

Se realizó un estudio descriptivo de la incidencia de brotes de gastroenteritis por Norovirus en el período del 15/10/2004 al 30/10/2005. La población del estudio fueron los residentes en Cataluña que en el periodo del estudio hubieran estado ingresados en algunos de los hospitales o residencias en los que se hubiera detectado un brote por norovirus.

El brote se definió como la presentación de dos o más casos de gastroenteritis por norovirus en el mismo centro en el que al menos uno de los casos hubiera estado confirmado microbiologicamente. Se realizó una encuesta epidemiológica para cada uno de los brotes investigados. Las variables del estudio fueron: número de expuestos, enfermos, tasa de ataque, mecanismo de transmisión, ámbito (centros sanitarios o residencias), región sanitaria, mes del año, y duración. También se realizó una encuesta epidemiológica a cada uno de los enfermos para recoger información demográfica, síntomas clínicos, duración de los síntomas, mecanismo de transmisión y resultado de las muestras clínicas. Mediante técnicas de enzimoimunoensayo y PCR se investigó la presencia de Norovirus en muestras clínicas de los afectados.

Se calculó la incidencia en cada uno de los brotes y la incidencia anual por centros en un año natural. Se utilizó como denominadores los pacientes censados en cada centro en el momento del estudio y el número de residencias y hospitales según el censo oficial del año 2005 respectivamente. Los intervalos de confianza se calcularon al nivel del 95\% según una distribución de Poisson. Se comparó la variable dependiente, ámbito de presentación (hospital o residencia), con el resto de varia- 
bles la odds ratio (OR) y su intervalo de confianza (CI). La existencia de diferencias estadísticamente significativas entre la variable dependiente (tipo de centro) y el resto de variables cualitativas del estudio se estudió con la prueba de $\chi^{2}$ y con las variables cuantitativas con la t de Student con un grado de significación (p) inferior a 0,05 .

\section{RESULTADOS}

Se detectaron 17 brotes, 6 en centros sanitarios $(35,3 \%)$ y 11 en residencias $(64,7 \%)$. La incidencia por centros fue de $2,1 \%$ (CI 95\% 1,3-3,3) y fue superior en hospitales en comparación a las residencias $(\mathrm{OR}=2,8$ IC 95\% 1,0-7,8) (tabla 1).

La mayoría de los brotes $76,5 \%(13 / 17)$ se presentaron entre el mes de octubre de 2004 y enero de 2005. El resto de brotes se presentaron en el mes de septiembre de
2005. Durante más de 6 meses no se detectó ningún brote en el conjunto de Cataluña (figura 1). Todas las regiones sanitarias registraron algún brote.

El 94,1\% (16/17) de los brotes se produjeron por transmisión persona a persona y sólo el 5,9\% (1/17) por alimentos. En todos los brotes se confirmó que el agente responsable fue Norovirus. El 92,8\% (13/14) fueron causados por el genogrupo II.4 y el $7,2 \%$ (1/14) por el II.b. En tres brotes no se pudo determinar el genogrupo. La media de días entre el primer y último caso del brote fue de 11,4 (DE =6,9), y fue superior en los centros sanitarios $(X=14,2 ; D E=9,7)$ en comparación a las residencias $(X=9,7$; $\mathrm{DE}=5,0)$, pero las diferencias no fueron estadísticamente significativas $(\mathrm{p}>0,05)$.

El censo total de personas expuestas en los centros con brotes fue de 1951 y la tasa de ataque global fue del 33,4\% (652/1951) y fue ligeramente superior en las residen-

Tabla 1

Incidencia, tasas de ataque y duración de los brotes en residencias y hospitales en Cataluña

\begin{tabular}{|c|c|c|c|}
\hline & Total & Hospital & Residencias \\
\hline \multicolumn{4}{|l|}{ Incidencia } \\
\hline Número de centros & 816 & 136 & 680 \\
\hline Número de brotes & 17 & 6 & 11 \\
\hline Incidencia anual $(\%)$ & 2,1 & 4,4 & 1,6 \\
\hline Incidencia anual IC 95\% & $1,3-3,3$ & $1,8-9,5$ & $0,9-2,9$ \\
\hline OR & - & 2,8 & 1,0 \\
\hline OR IC 95\% & - & $1,0-7,8$ & - \\
\hline \multicolumn{4}{|l|}{ Tasa de ataque } \\
\hline Pacientes expuestos & 1951 & 938 & 1013 \\
\hline Casos & 652 & 295 & 357 \\
\hline Tasa de ataque (\%) & 33,4 & 31,4 & 35,2 \\
\hline Tasa de ataque IC 95\% & $31,4-35,5$ & $28,6-34,5$ & $32,4-38,2$ \\
\hline OR & - & 1,0 & 1,2 \\
\hline OR IC $95 \%$ & - & - & $1,0-1,4$ \\
\hline \multicolumn{4}{|l|}{ Duración (días) } \\
\hline Número de brotes & 17 & 6 & 11 \\
\hline Media & 11,4 & 14,2 & 8,8 \\
\hline Desviación estándard & 6,9 & 9,2 & 5,6 \\
\hline Mediana & & 10,5 & 9,0 \\
\hline Rango & $1-28$ & $5-28$ & $1-18$ \\
\hline
\end{tabular}

OR, odds ratio; IC, intervalo de confianza. 
Figura 1

Distribución mensual de los brotes de Norovirus en Cataluña, 2004-2005

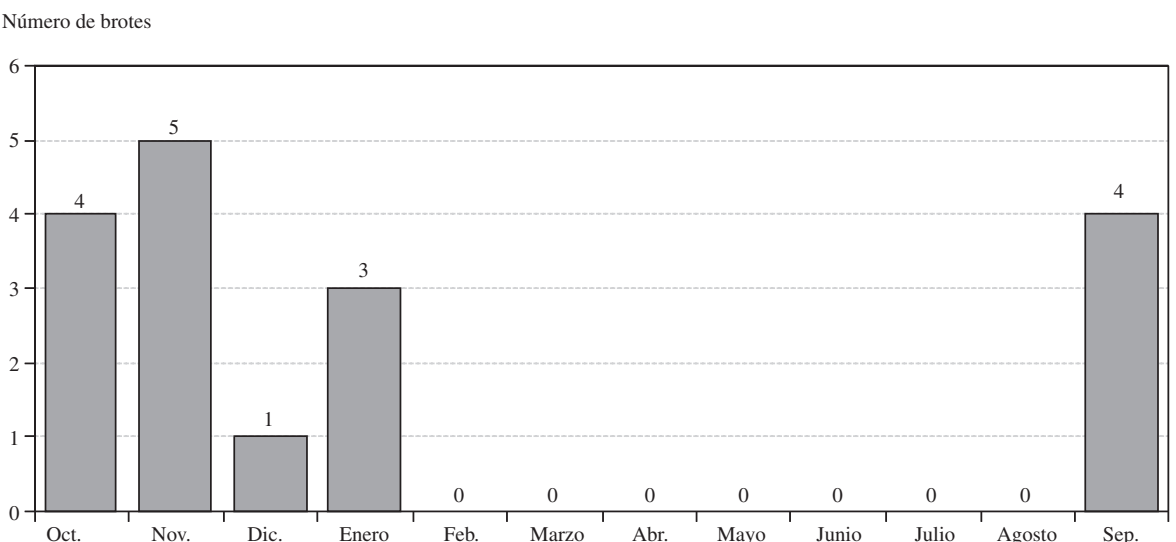

cias que en los hospitales (OR = 1,2 IC 95\% 1,0-1,4). El 18,5\% de los afectados fueron los propios trabajadores de los centros.

Se analizaron muestras de 246 pacientes y los resultados fueron positivos a Norovirus en $47,6 \%$ de los pacientes. La mayoría de los casos $(98,6 \%)$ se presentaron por transmisión persona a persona. Los síntomas más frecuentes fueron: diarrea $86,3 \%$, vómitos 55,2\%, dolor abdominal 37,3\%, náusea $33,0 \%$ y fiebre $17,0 \%$. La diarrea fue más frecuente en los pacientes de hospitales (tabla 2). La duración media de los síntomas

Tabla 2

Características de los pacientes con gastroenteritis por norovirus en brotes en residencias y hospitales en Cataluña

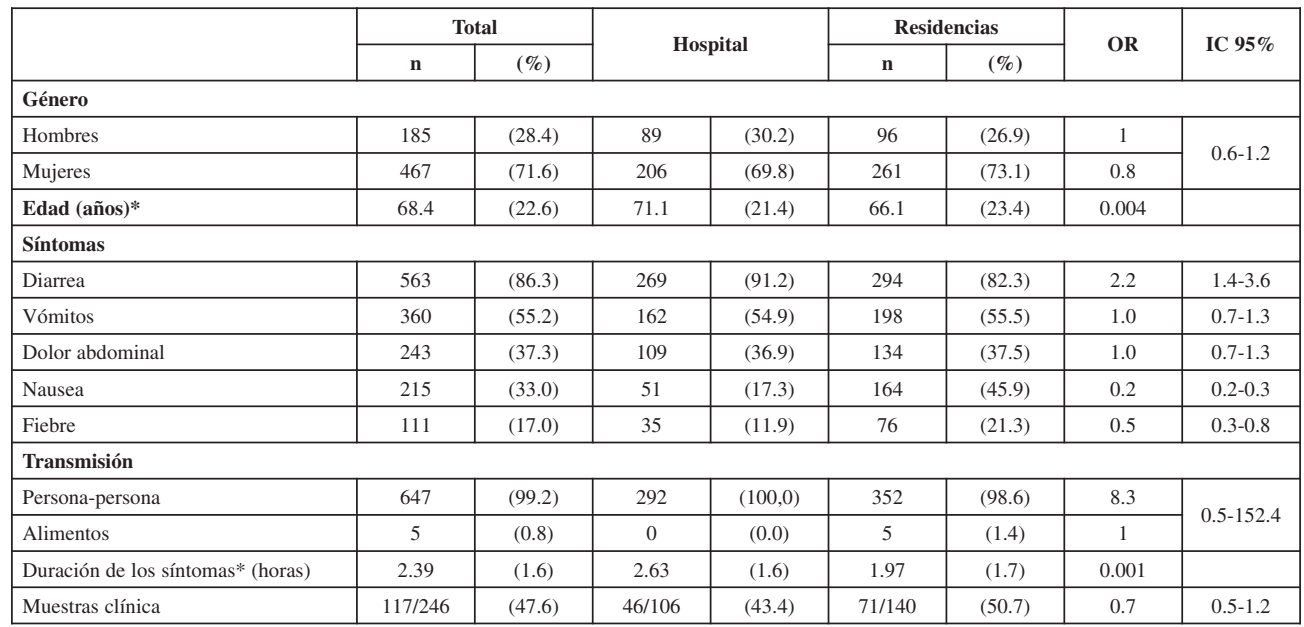

OR, odds ratio; IC, intervalo de confianza.

*Media y desviación estándard; 
fue de 2,39 dias $(\mathrm{SD}=1,6)$ y también fue superior en los pacientes hospitalizados 2,63 $(\mathrm{SD}=1,7)$ en comparación a los pacientes de residencias 1,97 $(\mathrm{SD}=1,7)(\mathrm{p}<0,0001)$.

\section{DISCUSIÓN}

La incidencia anual de brotes nosocomiales de Norovirus estimada por centros $(2,1 \%)$ se considera elevada y todavía fue superior en hospitales en comparación a las residencias. Para el conjunto de la población de Cataluña esto supone una incidencia de 0,2 brotes por $10^{5}$ hab, que se debe considerar superior a la estimada por Lopman et $\mathrm{al}^{9}$ para toda Europa, que fue de 0,3 a 0,7 brotes por $10^{5}$, pero teniendo en cuenta todos los tipos de brote.

Se debe señalar que la mayoría de los brotes se presentaron entre los meses de octubre y enero y que durante más de seis meses no se detectó ningún brote. La vigilancia de brotes en EE.UU ${ }^{10}$ y Europa ${ }^{11}$ muestra que la incidencia, la localización e incluso la estacionalidad pueden variar de una año para otro. Se ha discutido si los aumentos de brotes se deben a una mejoría de la vigilancia o a la emergencia de nuevas cepas. Dado que a lo largo del estudio se mantuvieron los mismos métodos de vigilancia, es probable que el aumento y posterior desaparición de los brotes se deba a la emergencia de una nueva cepa. Los dos genotipos detectados II.4 y II.b son los más frecuentes en Europa y ambos se ha asociado a cepas altamente recombinantes y emergentes ${ }^{12}$. Además, en nuestro país también podría existir un mayor riesgo de transmisión durante los meses fríos debido a la mayor hospitalización de las personas mayores y a que se vivan más horas en el interior de las viviendas lo cual puede implicar mayor grado de hacinamiento y mayores oportunidades para la transmisión.

La duración del cuadro clínico fue sólo 2,39 días en el conjunto de los pacientes, pero fue superior en los hospitalizados respecto a los de residencias. Otros estudios también han señalado una mayor gravedad y duración del cuadro clínico en pacientes afectados por otros procesos ${ }^{13}$. Como en otros brotes nosocomiales los trabajadores también resultaron afectados ${ }^{13}$, aunque las tasas de incidencia fueron inferiores a las del colectivo de pacientes ingresados.

La mayoría de brotes fueron causados por transmisión persona a persona y una vez que un centro resultó afectado las tasas de ataque final fueron elevadas tanto en residencias $(35,2 \%)$ como en los hospitales $(31,4 \%)$ lo que sugiere el bajo cumplimiento del lavado de manos ${ }^{15}$ y la dificultad del control de la transmisión debido a la diversidad de mecanismos de transmisión ${ }^{15}$. Las tasas de ataque fueron superiores en las residencias a las de los hospitales lo que sugiere una mayor transmisión del virus en las residencias y una menor aplicación de los protocolos para la prevención de la infección nosocomial.

La tasa de ataque global en los centros estudiados $(33,4 \%)$ se sitúa en valores intermedios a los descritos en la serie de Mattner et $\mathrm{al}^{16}$. El porcentaje de trabajadores afectados también es congruente con el descrito en otros estudios que señalan un riesgo alto de infección por norovirus en auxiliares de geriatría, enfermería y personal de limpieza, aunque inferior al de los propios pacientes ${ }^{17}$.

Las medidas de control esenciales son la desinfección de superficies con soluciones de hipoclorito a concentraciones de 1000 ppm, la prevención de la contaminación de los alimentos, la exclusión de los trabajadores enfermos, el aislamiento de cohortes de los pacientes y asegurar el lavado de manos de todos los trabajadores o el uso alternativo de soluciones alcohólicas ${ }^{17}$. Además se debe facilitar el acceso rápido a lavabos, uso de papel para el secado de manos y la disponibilidad de soluciones alcohólicas. Y 
lo más importante, se debe mantener un elevado cumplimiento del lavado de manos mediante actividades continuadas de educación sanitaria a los trabajadores ${ }^{18}$. Estudios recientes también señalan la dificultad de eliminar totalmente la transmisión en los colectivos afectados por lo que también se debe considerar la posibilidad de cerrar los colectivos y evitar la renovación de susceptibles hasta que se elimine totalmente la aparición de nuevos $\operatorname{casos}^{19}$.

\section{BIBLIOGRAFÍA}

1. Centers for Disease Control and Prevention. "Norwalk-like viruses": public health consequences and outbreak management. MMWR 2001;50(RR9):1-17.

2. Atmar RL, Opekum AR, Gilger MA, Estes MK, Crawford SE, Neill FH, Graham DY. Norwalk virus shedding after experimental human infection. Emerg Infect Dis 2008;14:1553-57.

3. García C, DuPont HL, Long KZ, Santos JI, Ko G. Asymptomatic Norovirus infection in Mexican children. J Clin Microbiol 2006;44:2997-3000.

4. Green YG, Belliot G, Taylor JL, Valdeuso J, Lew JF, Kapikian AZ, Lin FYC. A predominant role for Norwalk-like viruses as agents of epidemic gastroenteritis in Maryland nursing homes for the elderly. J Infect Dis 2002; 185:133-46.

5. Godoy P, Artigues A, Bartolomé R, Domínguez A, Plasencia A. Norovirus gastroenteritis outbreak by person to person transmisión in a nursing home. Med Clin (Barc) 2006;127(14):538-41.

6. Lopman BA, Andrews N, Sarangi J, Vipond IA, Brown DWG, Reacher MH. Institucional risk factors for outbreaks of nosocomial gastroenteritis: survival analysis of a cohort of hospital units in south-west England, 2002-2003. J Hosp Infect 2005; 60: 135-43.

7. Navarro G, Sala RM, Segura F, Arias C, Antón E, Varela P, et al. An outbreak of Norovirus infection in a long-term-care unit in Spain. Infec Control Hosp Epidemiol 2005;26:259-62.

8. Svraka S, Duizer E, Vennema H, de Bruin E, van der Veer B, Dorresteijn B, Koopmans M. Etiological role of viruses in outbreaks of acute gastroen- teritis in the Netherlands from 1994 through 2005. J Clin Microbiol 2007:45(5):1389-94.

9. Lopman BA, Reacher MH, Duijnhoven van Y, Hanon FX, Brown D, Koopmans M. Viral gastroenteritis outbreaks in Europe, 1995-2000. Emerg Infect Dis 2003;9:90-6.

10. Blantun LH, Adams SM, Beard RS, Wei G, Bulens SN, Widdowson MA, et al. Molecular and epidemiologic trends of calicivirus associated with outbreaks of acute gastroenteritis in the United Status, 2000-2004. J Infect Dis 2006;193:413-21.

11. Lopman BA, Reacher MH, Duijnhoven van Y, Hanon FX, Brown D, Koopmans M. Viral gastroenteritis outbreaks in Europe, 1995-2000. Emerg Infect Dis 2003;9:90-6.

12. Siebenga JJ, Vennema H, Duizer E, Koopmans PG. Gastroenteritis caused by Norovirus GGII.4, the Netherlands, 1994-2005. Emerg Infec Dis 2007;13(1):144-6.

13. Lopman BA, Reacher MH, Vipond IA, Sarangi J, Brown DWG. Clinical manifestation of Norovirus gastroenteritis in health care settings. CID 2004;39:318-24.

14. Wu HM, Fornek M, Schwab KJ, Chapin AR, Gibson K, Schawab E et al. A Norovirus outbreak at a long-term care facility: the role of environmental surface contamination. Infec Control Hosp Epidemiol 2005;26:802-9.

15. Cooper E, Blamey S. A Norovirus gastroenteritis epidemic in a long-term-care facility. Infec Control Hosp Epidemiol 2005;26:256-8.

16. Mattner F, Mattner L, Borck HU, Gastmeier P. Evaluation of the impact of the source (patient versus staff) on nosocomial norovirus outbreak severity. Infect Control Hosp Epidemiol. 2005;26(3):268-72.

17. Álvarez M del C. Norovirus: brotes en instituciones cerradas. En: Bellido Blasco LB, editor. Madrid: Epidemiología de las gastroenteritis agudas víricas. Sociedad Española de Epidemiología; 2007. p. 119-146.

18. Dolin R. Norovirus - Challenges to control. N Engl J Med 2007;357:1072-3.

19. Heijne JCM, Teunis P, Morroy G, Wijkman C, oostveen S, Duizer E, et al. Enhaced hygiene measures and norovirus transmission during an outbreak. Emerg Infect Dis 2009;15:24-30. 